\title{
OA04-04. Perceived parental willingness for their adolescents to participate in future HIV prevention trials: a survey conducted amongst adolescents in Soweto \\ K Otwombe*1, J Dietrich ${ }^{1}$, K Sikkema ${ }^{2}, \mathrm{G}$ de Bruyn ${ }^{1}, \mathrm{M}$ van der Watt ${ }^{1}$ and G Gray ${ }^{1}$
}

Address: ${ }^{1}$ Perinatal HIV Research Unit, Johannesburg, South Africa and ${ }^{2}$ Duke University, Durham, NC, USA

* Corresponding author

from AIDS Vaccine 2009

Paris, France. 19-22 October 2009

Published: 22 October 2009

Retrovirology 2009, 6(Suppl 3):O28 doi:I0.1 186/I742-4690-6-S3-O28

This abstract is available from: http://www.retrovirology.com/content/6/S3/O28

(C) 2009 Otwombe et al; licensee BioMed Central Ltd.

\section{Background}

Following the high HIV prevalence rates in Sub-Saharan Africa, several target specific interventions have been put in place with varying degrees of success. In implementing these interventions, adolescents have become a crucial target group. This study specifically examines perceived parental willingness for their adolescent to participate in HIV prevention trials by taking parent-adolescent communication into account.

\section{Methods}

The willingness to participate in future HIV prevention trials questionnaire and the Parent-Adolescent Communication Scale (PACS) formed part of a larger adolescent survey conducted among 16-18 year olds living in Soweto, an urban setting in Johannesburg, South Africa. Adolescent participants were interviewed about communication with parents or guardians regarding their sexual health. The questionnaires were interviewer administered.

\section{Results}

In total, 303 questionnaires were administered with a sample of $176(58.1 \%)$ females and $127(41.9 \%)$ males $($ median age $=17) .184(60.7 \%)$ participants had both parents alive, $78(25.7 \%)$ only the mother alive and 20 $(6.6 \%)$ only the father alive. Only $33(10.9 \%), 24$ (7.9\%) and $7(2.3 \%)$ participants indicated their mothers, fathers and guardians had post school training respectively. The PACS had a reliability of 0.77 with scores ranging between
7 and 28 with a median of 16 . A score greater than 14 was categorized as representing good communication skills. $266(88 \%)$ of the participants indicated their parents would be willing to allow them to participate in a future HIV vaccine trial. There was an association between parental willingness to participate and PACS scores ( $\mathrm{p}<$ $0.05, \mathrm{OR}=0.4085$, CI 0.2-0.8) in which univariate analysis showed that willingness was dependent on good communication scores $(\mathrm{OR}=2.4$, CI 1.2-5.0). The effect was particularly strong for females $(\mathrm{OR}=8.3$, CI $2.2-$ 30.7).

\section{Conclusion}

Perceived parental willingness for their adolescents to participate in future HIV vaccine trials is high and appears to be strengthened by good parent-adolescent communication. 